\title{
Engaging Patients in Healthcare Choices: Using the AHRQ Shared Decision Making Approach Eileene Shake, RN, DNP, NEA-BC
}

Eileene Shake*

University City of New York School of Professional of Services, USA

\begin{abstract}
According to a 2014 Institute of Medicine (IOM) Report the United States is increasingly burdened by factors that hamper delivering high-quality care to Americans during the end of life. The IOM report points out the importance of engaging patients and their families in shared decision making and advance care planning. Eighteen years ago nurse researchers informed us that if nurses, physicians, and health care planners knew more about the patient's healthrelated preferences, our society could reduce health care cost, provide a higher quality of effective care, and achieve the individual patient's goals related to their care. Today, it is imperative that healthcare providers and the entire healthcare interdisciplinary team engage patients in shared decision making when it comes to selecting treatment options. Engaging Patients in Healthcare Shared Decision Making: Three Major Areas of Choice
\end{abstract}

\section{Background}

According to a 2014 Institute of Medicine (IOM) Report the United States is increasingly burdened by factors that hamper delivering highquality care to Americans during the end of lifen [1]. The IOM report points out the importance of engaging patients and their families in shared decision making and advance care planning. Eighteen years ago nurse researchers informed us that if nurses, physicians, and health care planners knew more about the patient's health-related preferences, our society could reduce health care cost, provide a higher quality of effective care, and achieve the individual patient's goals related to their care [2]. Today, it is imperative that healthcare providers and the entire healthcare interdisciplinary team engage patients in shared decision making when it comes to selecting treatment options. Key findings and recommendations in the 2014 IOM report inform healthcare professionals, patients, payers, policy makers and caregivers of the importance of frequent clinician patient conversations about end-oflife care preferences, values, and goals [3].

\section{Problem - Lack of engaging patients in shared decision making}

Clinicians and other interdisciplinary team members providing chronic disease management care and palliative care need to increase initiating high-quality conversations about advance care planning. These conversations will lead to identifying the patients values, goals, theirpreferred path to a state of comfort, health, and sense of wellbeing. The patient's preference needs to be the driver when the interdisciplinary healthcare team assists the patient with making the best choice for treatment and care options.

Currently there is a problem in the nation as health care providers are not engaging frequently enough in conversations with patients and their family members using a shared decision making approach in advanced care planning, chronic disease management, or where the patient prefers to receive health care services. To compound this problem there are three major areas that lack attention in nursing and medical school curricula.

\section{These deficient areas include the following}

1. Lack of attention on shared decision making approaches in nursing and medical school curricula and according to the IOM report (2014) deficits in equipping physicians with sufficient communication skills exist.
2. The IOM report (2014) showed there is a lack of attention to palliative care in nursing and medical school curricula.

3. Kubler, Lampley, Shake, White-Hurst, Taggart et al. identified there is a lack of attention in nursing school curricula on managing chronic disease symptoms from conducting a systematic review of the literature to identify nursing curricula that include content on managing chronic disease symptoms [4].

Based on the lack of attention in the health care professionals education curricula on shared decision making, palliative care, and managing chronic disease symptoms one can see why they are not frequently engaging in shared decision making discussions with the patients and their family members. It also explains why we currently have a problem in this nation with the quality of outcomes in patients suffering from chronic disease, and the need for palliative care services.

\section{Three specific action steps to increase engaging patients in shared decision making}

Healthcare professionals can address these problems by taking 3 specific action steps. The first step includes health professionals assuming responsibility to educate themselves on the Agency for Healthcare Research and Quality (AHRQ) SHARED Decision Making Approach. The second step is for healthcare providers and educators to develop and implement community based educational programs for patients and the general population on shared decision making, chronic disease management, and palliative care. The third step is for healthcare educators and healthcare professional organizations to include shared decision making, managing chronic disease, and palliative care in the nursing and medical school curricula. *Corresponding author: Eileene Shake, University City of New York School of
Professional of Services, USA, E-mail: eshake@sc.rr.com

Received: April 26, 2016; Accepted: April 26, 2016; Published: May 03, 2016

Citation: Shake H (2016) Engaging Patients in Healthcare Choices: Using the AHRQ Shared Decision Making Approach Eileene Shake, RN, DNP, NEA-BC. J Pat Care 2: e102. doi: 10.4172/2573-4598.1000e102

Copyright: (c) 2016 Shake H. This is an open-access article distributed under the terms of the Creative Commons Attribution License, which permits unrestricted use, distribution, and reproduction in any medium, provided the original author and source are credited. 


\section{Three major areas for patient choice in shared decision making}

Three major areas of patient choice to consider when engaging in shared decision making include: treatment options on end of life care, chronic disease management, and their preferred setting to receive health care services. Providing patient education on treatment options using the AHRQ SHARED Decision Making tool kit and resources can help achieve the patient's goals and higher quality patient outcomes. Information on the tool kit and many other free resources can be found at http://www.ahrq.gov/professionals/education/curriculum-tools/ shareddecisionmaking/index.html. The SHARED Decision Approach has five steps that providers and patients can take to identify the best possible health care decisions [5].

\section{These include (AHRQ 2014)}

1. Seek your patient's participation

2. Help your patient explore and compare treatment options

3. Assess your patient's values and preferences

4. Reach a decision with your patient

5. Evaluate your patient's decision

Three questions that patients and the general population can be educated on to ask their providers include the following (AHRQ, 2014)

1. What are my treatment options?

2. What are the benefits and harms (risks)?

3. Where can I find more information to help me decide?

Patients can find more information at: http://www.ahrq.gove/ patietns-consumers/treatmentoptions.

\section{Settings for providing patient education on shared decision making}

Examples of settings where education on shared decision making and provision of health care services could occur include, providers offices, hospitals, kidney dialysis centers, community clinics, the patients home, community centers, places of worship, senior centers, and naturally occurring retirement communities. An example of a Nurse Practitioner led transition care clinic that provides chronic disease management is located in Moore County, North Carolina and is the FirstHealth Transition Care Clinic. According to Batchelor, this clinic focuses on filling the gap between hospital-to-home or emergency department-to-home for individuals with chronic diseases who do not have a primary care provider or other resources [6]. More information on this clinic can be found on their website at: https://www.firsthealth. org/specialties/primary-care-specialty-clinics/transition-care-clinics.

The First Health Transition Care Clinic has a team of diverse professionals providing services Batchelor, and any of these team members could engage the patient in shared decision making. Chaplains, ministers, parish nurses and other faith leaders can engage patients in shared decision making. Physicians, nurses, hospital case managers, nurse practitioners, social workers, care coordinators, care managers, discharge planners, and providers' office staff can engage patients in shared decision making.

\section{Summary}

Healthcare professionals, regulatory agencies, policy makers, health insurers, social workers, and other members of the interdisciplinary health teams that are providing care to patients with chronic disease symptoms, palliative care services and end-of -life care need to engage patients and their family members in shared decision making. It is imperative to take action steps to increase engaging patients in shared decision making as the Institute of Medicine, U.S. Department of Health and Human Services, and the Centers for Medicare and Medicaid are engaged in initiates to address the escalating population of patients who are living with chronic symptomatic diseases Kuebler, Lampley, Shake, et al. (2014)

With a rapidly rising American population of aging baby boomers with multiple chronic diseases and end-of-life care needs, we must take action to reduce care costs and improve the quality outcomes for these patients. Providing palliative care services and chronic disease symptom management with the patient making a choice in the treatment and place of service can improve patient outcomes and reduce cost. Engaging patients and letting them know they have choices and can share in the decisions on their treatment options and desired place of receiving care can lead to reducing pain and suffering, improve patients' quality of life, and reduce healthcare costs.

\section{References}

1. National Academices of Science (2014) Dying in America: Improving quality and honoring individual preferences near the end of life.

2. Brennan P F, Strombom I (1998) Improving Health Care by Understanding Patient Preferences: The Role of Computer Technology. Journal of the American Medical Informatics Association : JAMIA, 5: 257-262.

3. National Academices of Science (2014) Dying in America: Key findings and recommendations.

4. Kuebler K, Lampley T, Shake E, White-Hurst E, Taggart, et al. (2014) A systematic review: A collaborative partnership on evaluating graduate nursing education in chronic symptomatic disease. Clinical Scholars Review, 7: 98-104.

5. Agency for Health Care Research and Quality (2015) The SHARE Approach.

6. Batchelor C (2015) Nurse practitioner-led transition care clinic- A new perspective on chronic disease management. Tarheel Nurse, 77: 1-18. 